\title{
Extraction of Tubular Structures over an Orientation Domain
}

\author{
Mickaël Péchaud \\ DI \\ Renaud Keriven \\ CERTIS \\ École Normale Supérieure \\ France \\ École des Ponts \\ France \\ Gabriel Peyré \\ CEREMADE \\ Université Paris Dauphine \\ France
}
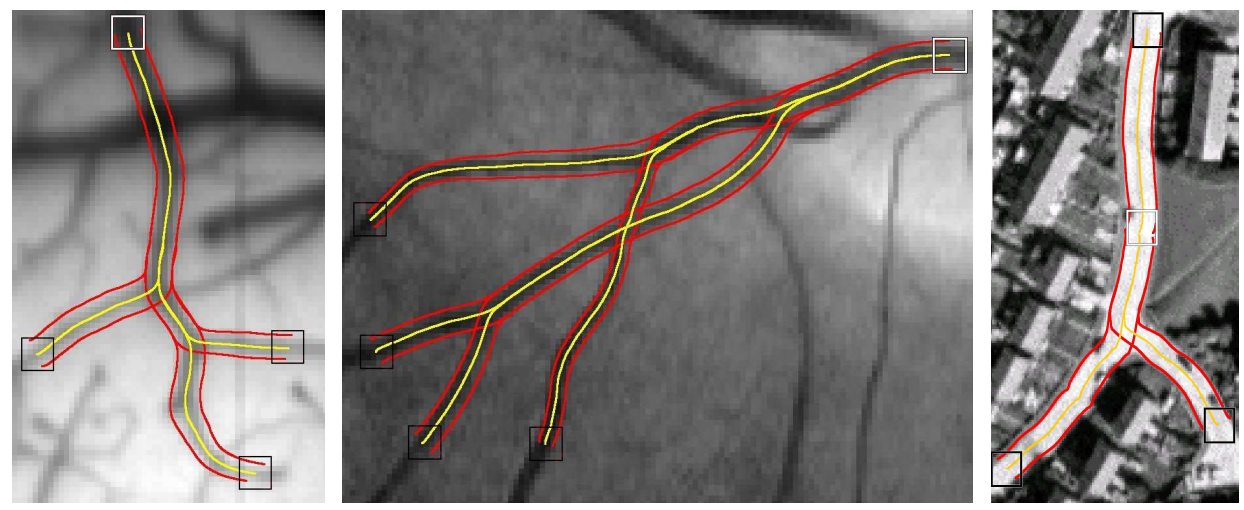

Figure 1. Centerlines and radii extraction of vessels in a retinal image, vessels in a cortical image and roads in a satellite image.

\begin{abstract}
This paper presents a new method to extract tubular structures from bi-dimensional images. The core of the proposed algorithm is the computation of geodesic curves over a four-dimensional space that includes local orientation and scale. These shortest paths follow closely the centerline of tubular structures, provide an estimation of the radius and can deal robustly with crossings over the image plane. Numerical experiments on a database of synthetic and natural images show the superiority of the proposed approach with respect to several method based on shortest paths extractions.
\end{abstract}

\section{Introduction}

The problem of tubular structures extraction has received considerable attention in the computer vision and medical imaging communities - see for instance the recent review by Kirbas and Quek [11]. They generally rely on the use of a local detector, postprocessed by a method that links locally detected tubular structure.

Various methods have been developed to locally detect tubular structures, for instance thresholding, ridge or crest detection[1, 17], wavelets [9], and matching filters [8, 2].

Many processes allow to link or post-process the locally detected points. Besides classical fusion processes [3, 25] and region growing algorithm [7, 21], tracking-based approaches such as $[18,13,20]$ are an important attempt to to address this issue. They are based on a propagation inside the tubular structure from a known starting point by using a local search.

The notion of shortest path has proved to be efficient in order to perform global segmentation of tubular structures, in images, see for instance [5, 16]. These geodesic curves can also be used to extract tubular structure centerlines, as proposed by Deschamps and Cohen [6] and by Santamaria et al.[19].

In order to enhance geodesic-based methods, $\mathrm{Li}$ and Yezzi [14] have proposed to extend the shortest path computation to a higher dimensional domain. They include the local radius of the vessels as an additional scale dimension in order to stabilize the computations and to select the centerline without any post-processing. This method is however facing difficulties on 2D images because of the design of their local vessel detector and of the junctions that are not handled in this 2D+1D space and scale domain.

This paper shows how to robustly detect tubular structures in 2D images by the use of shortest paths. It intro- 
duces an additional local orientation dimension that disambiguates the crossing singularities using pattern-recognition inspired techniques. Centerline detection and radius estimation are completely embedded in the proposed segmentation method and does not rely on additional properties of the images (such as centerlines being the darker part of vessels), nor on a post-processing step. Numerical results on synthetic and real-life examples shows the advantage of using this orientation information. The database will available online so that future method can compare their results to the proposed approach.

\section{Shortest paths in orientation domain}

This paper considers a gray scale image $I:[0,1]^{2} \rightarrow$ $[0,1]$ and in numerical computation, this image is sampled regularly on a grid of $n \times n$ pixels. In the following derivative operators in the continuous domain are used although the computations are done on the discrete grid using finite differences.

\subsection{Local Tubular Structure Model}

At the core of the proposed approach is the local detection of tubular structures. This paper uses a normalized cross-correlation with a tubular model as a local feature indicator.

The local geometry of a tubular structure is captured with a model $M(x) \in \mathbf{R}$ for $x=\left(x_{1}, x_{2}\right) \in \Lambda=$ $\left[-\Lambda_{1}, \Lambda_{1}\right] \times\left[-\Lambda_{2}, \Lambda_{2}\right]$. The models considered is this article are of the form $M\left(x_{1}, x_{2}\right)=m\left(x_{2}\right)$ and therefore only depend on a $1 \mathrm{D}$ profile $m$. A model is thus a small template which represents a typical horizontal tubular structure of normalized width one wishes to detect. This normalized pattern is then rotated and scaled in order to define warped models $M_{r, \theta}(x)$ for $x \in \Lambda(r, \theta)=r R_{\theta}(\Lambda)$

$$
\forall x \in \Lambda(r, \theta), \quad M_{r, \theta}(x) \stackrel{\text { def. }}{=} M\left(R_{-\theta}(x / r)\right)
$$

where $R_{\theta}$ is the planar rotation of angle $\theta$. Each pattern $M_{r, \theta}$ looks like a typical tubular structure oriented along direction $\theta \in[0, \pi)$ and of width $r>0$.

The model $M$ can be tuned for each targeted application. Figure 2 shows some examples of these patterns for various $r$ and $\theta$.

The choice of $\Lambda$ is a trade-off between robustness and accuracy of local structure detector. Experiments where carried out to determine an "optimal" $\Lambda$, which will not be detailled in this article due to the lack of space. $\Lambda$ was chosen as a vertical rectangle of dimension $1 \times 2$ (cf. figure 2 ). The experiments show low sensitivity of the proposed method w.r.t. this choice.

Model for road extraction. A typical road in satellite imaging has a slow variation of intensity along a curve. It is

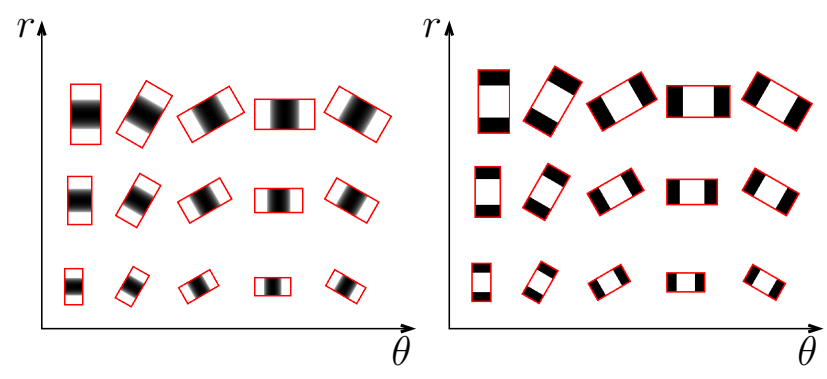

Figure 2. Vessel model (left) and road model (right) for different orientations and scales.

thus efficiently captured by a binary model defined as

$$
m\left(x_{2}\right) \stackrel{\text { def. }}{=} \begin{cases}0 & \text { for }\left|x_{2}\right|>\Lambda_{2} / 2 \\ 1 & \text { otherwise. }\end{cases}
$$

Model for blood vessels. Two important applications of vessels extraction are cortical optical imaging [12] and retinal imaging [15]. In order to capture efficiently blood vessels in these medical images, one designs a specific local pattern derived from a physical model of these structures. The major difference with road tracking is that a vessel is not of constant intensity across its section.

The model is defined as

$m\left(x_{2}\right) \stackrel{\text { def. }}{=} \begin{cases}1 & \text { for }\left|x_{2}\right|>\Lambda_{2} / 2 \\ \exp \left(-\alpha \sqrt{(1 / 2)^{2}-\left(x_{2} / \Lambda_{2}\right)^{2}}\right) & \text { otherwise }\end{cases}$

This model assumes that the image has some basal intensity outside the vessels - e.g. light is reflected without absorption. For a point inside a vessel of circular section, the intensity is assumed to result from a light absorption (with coefficient $\alpha$ ) proportional to the vessel width at this point.

\subsection{Orientation Domain Lifting}

In order to extract tubular structures that might exhibit self-crossings, the 2D image $I$ is lifted to a $4 \mathrm{D}$ representation $F$

$$
F: \Omega \stackrel{\text { def. }}{=}[0,1]^{2} \times\left[r_{\min }, r_{\max }\right] \times[0, \pi) \longmapsto \mathbb{R}
$$

defined as the normalized cross-correlation between the image and the local model introduced in equation (1)

$\forall(x, r, \theta) \in \Omega, F(x, r, \theta) \stackrel{\text { def. }}{=} N C C_{\Lambda(r, \theta)}\left(M_{r, \theta}(\cdot), I(x+\cdot)\right)$

where $I(x+\cdot)$ is the image translated by $x, N C C_{A}(f, g)$ is the normalized cross-correlation between $f$ and $g$ over the domain $A$, defined by:

$$
N C C_{A}(f, g) \stackrel{\text { def. }}{=} \frac{\int_{A}(f-\bar{f})(g-\bar{g})}{\sqrt{\int_{A}(f-\bar{f})^{2}} \sqrt{\int_{A}(g-\bar{g})^{2}}}
$$


where $\bar{h}=\frac{\int_{A} h}{\mu(A)}, \mu(A)$ being the area of $A$.

Function $F$ ranges from -1 to 1 , is invariant under local intensity changes and is an indicator of how likely a tubular structure of radius $r$ and orientation $\theta$ is present at location $x$.

The main interest of this lifting $I \mapsto F$, as already noticed in [10], is that it disambiguates situations where two tubular structures are crossing each other. Figure 3 shows an example of a medical image where orientation lifting is crucial to distinguish locally between two orientations.
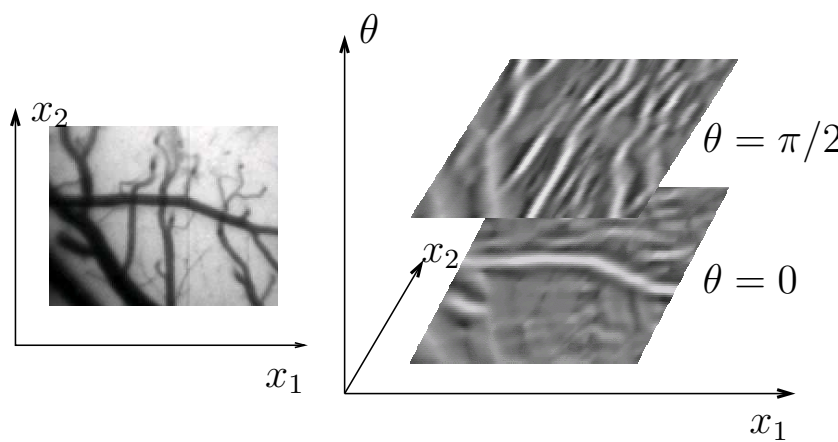

Figure 3. left: original 2D image. right: 4D lifting (fixed radius), ranging from -1 (black) to 1 (white).

This lifting is computed for $n_{r}$ radii evenly spaced in $\left[r_{\min }, r_{\max }\right]$ and $n_{\theta}$ orientations evenly spaced in $[0, \pi)$, with $n_{r}=12$ and $n_{\theta}=12$ in the experiments. This requires $O\left(\left(r_{\max } n\right)^{2} n^{2} n_{r} n_{\theta}\right)$ operations with $r_{\max } \ll 1$ and $n_{r}, n_{\theta} \ll n$.

The 4D lifting (5) defines an isotropic metric $\rho$ over the 4D domain $\Omega$

$$
\forall \omega \in \Omega, \quad \rho(\omega) \stackrel{\text { def. }}{=} \max (1-F(\omega), \varepsilon) .
$$

The parameter $\varepsilon$ prevents the metric to vanish and is set to $\varepsilon=10^{-3}$ in the numerical tests.

\subsection{Distance Map and Geodesic Computation}

The length of a lifted curve $\gamma:[0,1] \rightarrow \Omega$ over the lifted domain is defined as

$$
\mathcal{L}_{F}(\gamma) \stackrel{\text { def. }}{=} \int_{0}^{1} \rho(F(\gamma(t)))\left\|\gamma^{\prime}(t)\right\| \mathrm{d} t
$$

is

The length of the speed vector $v=\gamma^{\prime}(t)=\left(v_{x}, v_{r}, v_{\theta}\right)$

$$
\|v\|^{2} \stackrel{\text { def. }}{=} v_{x}^{2}+\lambda v_{r}^{2}+\mu v_{\theta}^{2} .
$$

where $(\lambda, \mu)$ are normalizing constants set to $\lambda=0.5$ and $\mu=0.1$ in numerical experiments. In practice one experiments strong robustness with respect to the choice of $(\lambda, \mu)$.

Given a set $\mathcal{A} \subset \Omega$ of seeds points and a set $\mathcal{B} \subset \Omega$ of ending points, a shortest lifted curve $\gamma^{*}(t) \subset \Omega$ joining $\mathcal{A}$ to $\mathcal{B}$ is defined as a shortest path for the metric $\mathcal{L}_{F}$

$$
\gamma^{*}(\mathcal{A}, \mathcal{B}) \stackrel{\text { def. }}{=} \underset{\gamma \in \pi(\mathcal{A}, \mathcal{B})}{\operatorname{argmin}} \mathcal{L}_{F}(\gamma)
$$

where $\pi(\mathcal{A}, \mathcal{B})$ is the set of curves $\gamma$ such that $\gamma(0) \in \mathcal{B}$ and $\gamma(1) \in \mathcal{A}$. The corresponding geodesic distance is $d_{F}(\mathcal{A}, \mathcal{B})=\mathcal{L}_{F}\left(\gamma^{*}\right)$.

In practice, $\gamma^{*}$ is estimated as follows [23]: the distance to the seeds $\mathcal{A}$ is the geodesic action map $\mathcal{U}_{\mathcal{A}}(\omega)=$ $d_{F}(\mathcal{A}, \omega)$ is the unique viscosity solution of the Eikonal equation

$$
\begin{gathered}
\left\|\nabla \mathcal{U}_{\mathcal{A}}(\omega)\right\|=\rho(\omega), \quad \text { with } \quad \forall \omega \in \mathcal{A}, \mathcal{U}_{\mathcal{A}}(\omega)=0 \\
\text { where } \quad \nabla \mathcal{U}_{\mathcal{A}}=\left(\frac{\partial \mathcal{U}_{\mathcal{A}}}{\partial x}, \lambda \frac{\partial \mathcal{U}_{\mathcal{A}}}{\partial \theta}, \mu \frac{\partial \mathcal{U}_{\mathcal{A}}}{\partial r}\right)^{\mathrm{T}},
\end{gathered}
$$

and where one has to be careful about computing the derivatives with respect to $\theta$ modulo $\pi$.

Calling $\omega_{1} \in \mathcal{B}$ the point in $\mathcal{B}$ with lowest distance to $\mathcal{A}$, the geodesic curve $\gamma^{*}$ between $\mathcal{A}$ and $\mathcal{B}$ is then obtained by a gradient descent of $\mathcal{U}_{\mathcal{A}}$

$$
\frac{\mathrm{d} \gamma^{*}}{\mathrm{~d} t}(t)=-\nabla \mathcal{U}_{\mathcal{A}}\left(\gamma^{*}(t)\right) \quad \text { with } \quad \gamma(0)=\omega_{1}
$$

The numerical computation of $\mathcal{U}_{\mathcal{A}}$ on a discrete grid of $N \stackrel{\text { def. }}{=} n^{2} n_{r} n_{\theta}$ pixels is done in $O(N \log (N))$ operations with the Fast Marching algorithm of Sethian [22]. Furthermore, the Fast Marching is stopped as soon as the set $\mathcal{B}$ is reached, leading to important computation savings. During the Fast Marching computation, one should treat the orientation dimension $\theta \in[0, \pi)$ with periodic boundary conditions $0 \simeq \pi$.

\section{4D Curves Extraction}

\subsection{Shortest Paths and 4D curves}

In order to extract a $4 \mathrm{D}$ curve $c\left(x, x^{\prime}\right)$ between two points $x, x^{\prime} \in[0,1]^{2}$, one needs to compute a shortest lifted tubular structure $\gamma^{*}$ between the lifted points in $\Omega$ that correspond to the $x_{i}$. Let $\mathcal{A}(x)$ and $\mathcal{A}\left(x^{\prime}\right)$ be defined by

$$
\mathcal{A}\left(x^{\left({ }^{\prime}\right)}\right) \stackrel{\text { def. }}{=}\left\{\left(x^{\left({ }^{\prime}\right)}, r, \theta\right) \backslash r \in\left[r_{\min }, r_{\text {max }}\right], \theta \in[0 ; \pi)\right\} .
$$

This $4 \mathrm{D}$ curve is then defined

$$
c_{x, x^{\prime}} \stackrel{\text { def. }}{=} \underset{\gamma \in \pi\left(\omega, \omega^{\prime}\right), \omega^{(\prime)} \in \mathcal{A}\left(x^{(\prime)}\right)}{\operatorname{argmin}} \mathcal{L}(\gamma) .
$$

This 4D curve contains three components $c_{x_{1}, x_{2}}(t)=$ $(\tilde{x}(t), r(t), \theta(t))$. The path $\tilde{x}(t) \subset[0,1]^{2}$ is the actual centerline over the image plane, whereas $r(t)$ and $\theta(t)$ give the local width and orientation of the tubular structure (figure 4), which can be important in medical application, for instance to compute the local blood flow or to detect tissue diseases. 


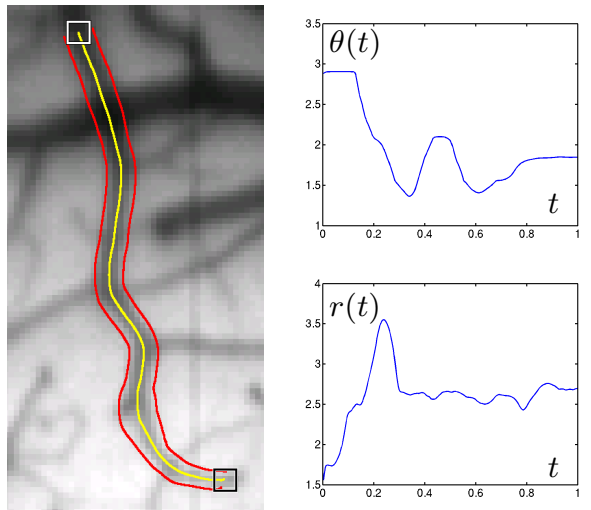

Figure 4. Left: extraction of a vessel in a cortical image. Starting point: white square. Ending point: black square. Right: corresponding orientation $\theta(t)$ and radius $r(t)$.

\subsection{Numerical Experiments}

\subsubsection{Phantom experiments.}

Experiments were carried out on several phantoms images, for which the centerlines positions and the radii have known analytical forms with sub-pixelic accuracy. The cross section of these phantoms corresponds to the model (3) with parameter $\alpha=0.01$. An additive Gaussian white noise with various amplitudes are added to the phantoms. Ten phantoms are generated for each condition, and each noise level, see Figure 5. In each case, the true starting and ending points of each phantom are used, as well as the true starting and ending radii for the [14] method. The resulting benchmark database will be available online in order to allow comparison with future works.
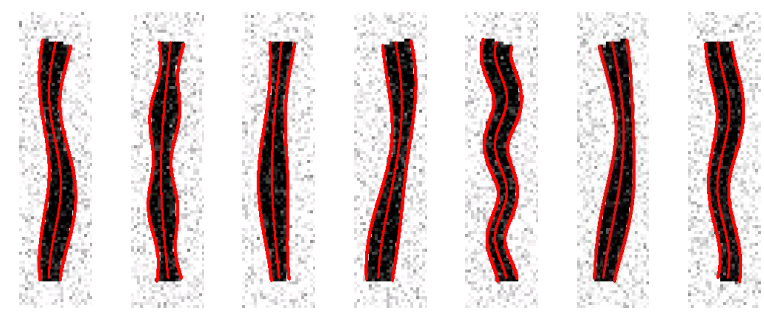

Figure 5. Some of the sample phantoms used as benchmarks (basic intensities range from 0 to 1 ), shown here with a spatially independent Gaussian noise of variance 0.15.

For each phantom and level of noise, three algorithms were tested: the classical 2D method Fast Marching [4] with pre-smoothing of the image (pre-smoothing is classically used in Fast Marching based methods to obtain a centered path), Li/Yezzi method[14] and the method proposed in this article. The smoothing parameter of the classical 2D method, the smoothing parameter as well the three more parameters of the 3D method were optimized for this benchmark. In order not to take advantage of the exact knowledge of the profile, the proposed method was applied with a vol- untary wrong model parameter $\alpha=0.1$. In each case, the true starting and ending points of each phantom are used, as well as the true starting and ending radii for the [14] method.

For each method, the error of the vessels centerline position and radius w.r.t the ground truth was computed using the following formula.

$$
\operatorname{Error}_{C}(c)^{2}=\int_{0}^{1}\left\|\tilde{x}(t)-\tilde{x}^{*}\left(t^{*}\right)\right\|^{2} \mathrm{~d} t
$$

where $t^{*}$ is such that $\tilde{x}^{*}(t)$ is the ground truth centerline point closest to the computed centerline $\tilde{x}(t)$.

The error of the radii estimation ([14] and proposed method only) was computed using the following formula:

$$
\operatorname{Error}_{R}(c)^{2}=\int_{0}^{1}\left|r(t)-r^{*}\left(t^{*}\right)\right|^{2} \mathrm{~d} t
$$

where $r$ is the radius computed by the method, and $r^{*}$ the ground truth radius.

Figure 6 shows $\operatorname{Error}_{C}(c)$ and $\operatorname{Error}_{R}(c)$ curves for several synthetic images as a function of the noise level. Using the 3D space+scale lifting [14] produces results of varying quality, and requires a careful tuning for each noise level of the parameter to achieve the optimal error rate. [4] with an optimal smoothing provides a precise evaluation of the centerline locations, but without any evaluation of the local radius. The proposed method provides both positions and radii with more robustness and accuracy.

\subsubsection{Real images experiments.}

Figure 1 shows results of tubular structure extraction for the three modalities considered in this paper:

- Left: vessels extraction on a retinal image from the DRIVE database [24, 15].

- Center: vessels extraction for a complex optical imaging of the cortex with several branches and intersections.

- Right: road extracted from a satellite image over an urban area.

The starting area $\mathcal{A}$ is shown with a white square and several end points $\omega$ are shown with black squares.

The crossings in the retinal image (left) show the interest of the 4D lifting. Note also the large overlap of the centerlines computed from several different points, the subpixelic precision of the centerline and the correct handling of intersections on the cortical image (center). Another interest of this method is that it automatically computes the radius $r(t)$ and orientation $\theta(t)$ parameters.

The precision of the 4D lifting method is evaluated on the DRIVE database [24, 15]. Approximate ground truth centerlines positions and radii are computed from the binary masks available with the database. Figure 7, shows 

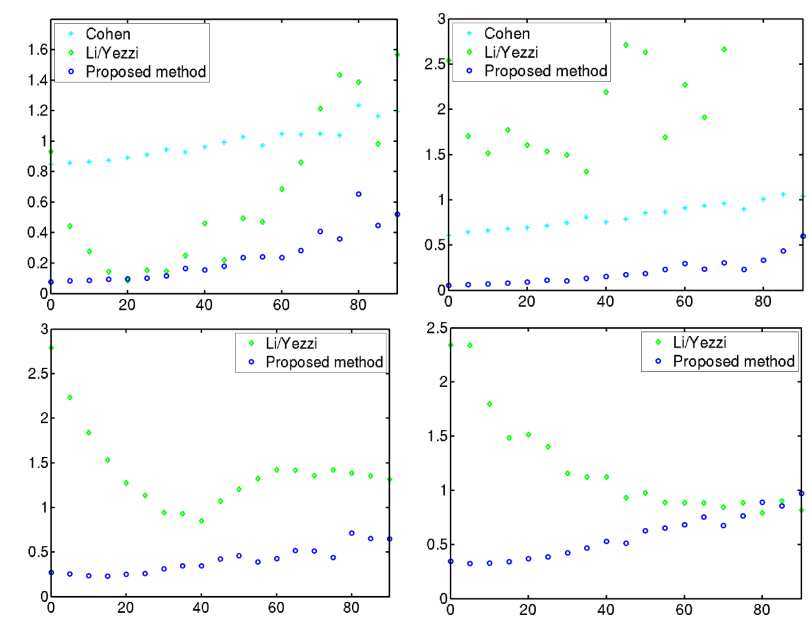

Figure 6. Centerlines and radii estimation for the first 2 phantoms presented fig. 5.

Top row : error $\operatorname{Error}_{C}(\gamma)$ (in pixel) between the computed and theoretical centerlines for the four methods versus noise level ( $100 \sigma$ where $\sigma$ is the independent Gaussian noise variance) (circles: proposed method, diamonds: Li/Yezzi[14], crosses: 2D Fast Marching with pre-smoothing[4] )

Bottom row : error $\operatorname{Error}_{R}(\gamma)$ (in pixel) for the two first methods versus noise ratio.

the binary segmented vessels together with the ground trust centerline position and boundaries (top and middle), as well as the result of the 4D lifting method (bottom).
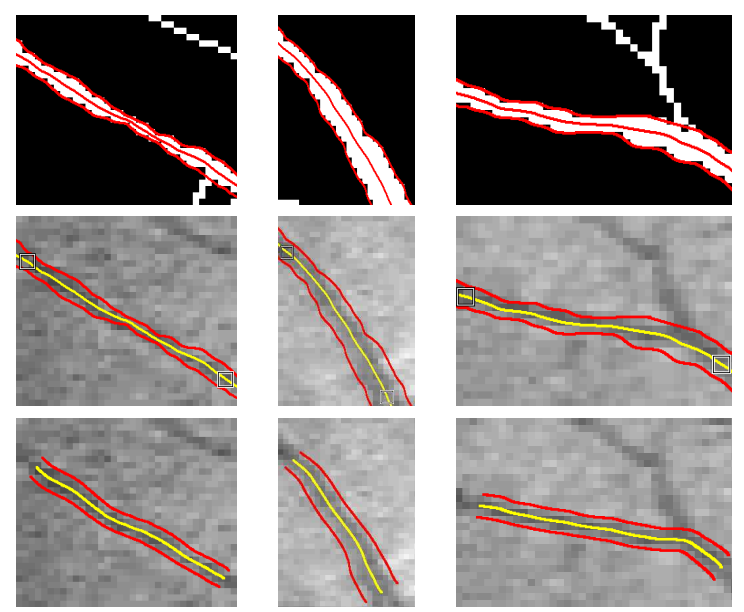

Figure 7. binary segmented images from the DRIVE database, together with the extracted ground trust. middle: corresponding images with ground-truth centerline and boundary and initial and ending points. bottom: centerlines positions and boundaries computed with the proposed method.

The three geodesic extraction algorithms are applied to these three images between the indicated starting and ending points. Table 1 report the centerlines position and radii errors $\operatorname{Error}_{C}(\gamma)$ and $\operatorname{Error}_{R}(\gamma)$ for each method.
For the centerline extraction, due to the lack of precision of the ground truth, there is no significant difference between the proposed 4D lifting method and the space only geodesic extraction with smoothing of the metric. The 3D space+scale lifting [14] method showed unstable behavior with respect to its initialization and parameters, which had to be chosen carefully - for the second image, no parameters giving a correct result were found. The $4 \mathrm{D}$ lifting method is also more precise for the radii estimation than the $3 \mathrm{D}$ lifting.

\begin{tabular}{|c|c|c|c|c|c|c|}
\hline & \multicolumn{2}{|c|}{ DRIVE 1 } & \multicolumn{2}{c|}{ DRIVE 2 } & \multicolumn{2}{c|}{ DRIVE 3 } \\
\hline & $\operatorname{Err}_{C}$ & $\operatorname{Err}_{R}$ & $\operatorname{Err}_{C}$ & $\operatorname{Err}_{R}$ & $\operatorname{Err}_{C}$ & $\operatorname{Err}_{R}$ \\
\hline 2D & 0.40 & - & 0.38 & - & 0.30 & - \\
\hline 3D & 1.33 & 1.67 & 3.13 & 3.31 & 0.53 & 1.90 \\
\hline 4D & 0.31 & 0.43 & 0.35 & 0.44 & 0.40 & 0.47 \\
\hline
\end{tabular}

Table 1. Centerlines positions Error $_{C}$ and radii Error estimation $_{R}$ errors on retina images for the three different methods.

\subsubsection{Sensibility of the proposed method with respect to the tubular structure model.}

In order to show that the proposed method in not sensitive to the choice of the tubular structure model, some tests were performed with different values of $\alpha$, showing that the proposed method is able to recover with good accuracy the centerlines and radii, even if the vessel model is unknown. Table 2 shows the results for one of these tests: for phantoms generated with $\alpha=0.01$, estimation with different fixed values of $\alpha$ are conducted for different noise levels. The obtained average errors remains comparable and in any case better than with [14] method.

\begin{tabular}{|c|c|c|c|c|}
\hline & \multicolumn{2}{|c|}{ Phantom 1 } & \multicolumn{2}{c|}{ Phantom 2 } \\
\hline & Error $_{C}$ & Error $_{R}$ & Error $_{C}$ & Error $_{R}$ \\
\hline$\alpha=0.01$ & 0.09 & 0.24 & 0.10 & 0.23 \\
\hline$\alpha=0.1$ & 0.09 & 0.24 & 0.11 & 0.31 \\
\hline$\alpha=1$ & 0.10 & 0.53 & 0.08 & 0.60 \\
\hline
\end{tabular}

Table 2. Centerlines positions Error $_{C}$ and radii $_{\text {Error }}$ estimation errors for two different vessel models (gaussian noise with $\sigma=$ $0.2)$

\subsubsection{Advantage of 4D modelling.}

As already noted, figure 1 (left) shows a crossing situation that cannot be handled by methods that do not take orientation into account. In the case of vessel extraction, going straightforward is much more probable when encountering a crossing. Figure 8 shows a synthetic example with a such simple crossing configuration. Both classical 2D Fast Marching and Li/Yezzi methods give an undesired path, while the proposed method finds a correct result. 
Figure 9 shows a real-life example with a simple road crossing. In this experiment, two starting points $x_{1}$ and $x_{2}$ were provided - e.g. the set of seed points is $\mathcal{A}\left(x_{1}\right) \cup \mathcal{A}\left(x_{2}\right)$. Shortest paths from two different ending points are shown for the three tested algoritms. The proposed method again finds the correct paths.

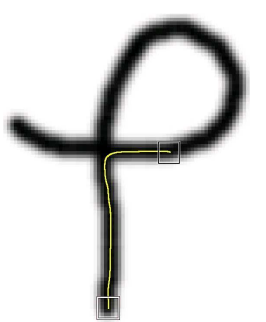

Classical FM

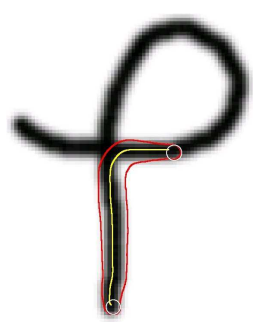

$\mathrm{Li} /$ Yezzi

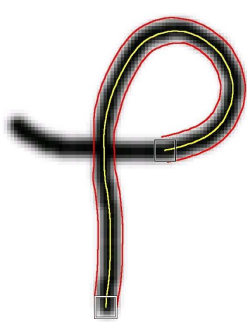

Proposed method
Figure 8. Comparison of the different methods when encountering a crossing. Only the proposed method goes from the start to the end point following the natural path for vessel extraction.

\section{Acknowledgments}

Left blank during the reviewing process

\section{Conclusion}

This article introduces a novel framework for the automatic extraction of tubular structures, estimating both centerlines and radii. Examples on synthetic, medical and satellite images, with potentially intersecting curves, show the necessity to incorporate the orientation information in the model. The proposed method was compared to state of the art methods, proving to be more accurate and more stable. It shows robust results on different types of images. Its independence with respect to the local tubular structures detector makes it adaptable to any modality. A synthetic dataset will be available online in order to establish a benchmark for future contributions on centerlines/radii extraction.

\section{References}

[1] S. Aylward, S. Pizer, D. Eberly, and E. Bullitt. Intensity ridge and widths for tubular object segmentation and description. In Proceedings of the 1996 Workshop of MMBIA '96, page 131, Washington, DC, USA, 1996. IEEE Computer Society. 1

[2] H. E. Bennink, H. C. van Assen, G. J. Streekstra, R. ter Wee, J. A. E. Spaan, and B. M. ter Haar Romeny. A novel 3d multi-scale lineness filter for vessel detection. In N. Ayache, S. Ourselin, and A. J. Maeder, editors, Proceedings of MICCAI (2), volume 4792 of Lecture Notes in Computer Science, pages 436-443. Springer, 2007. 1

[3] J. Canny. A computational approach to edge detection. IEEE Transactions Pattern Analysis and Machine Intelligence, 8(6):679-698, November 1986. 1

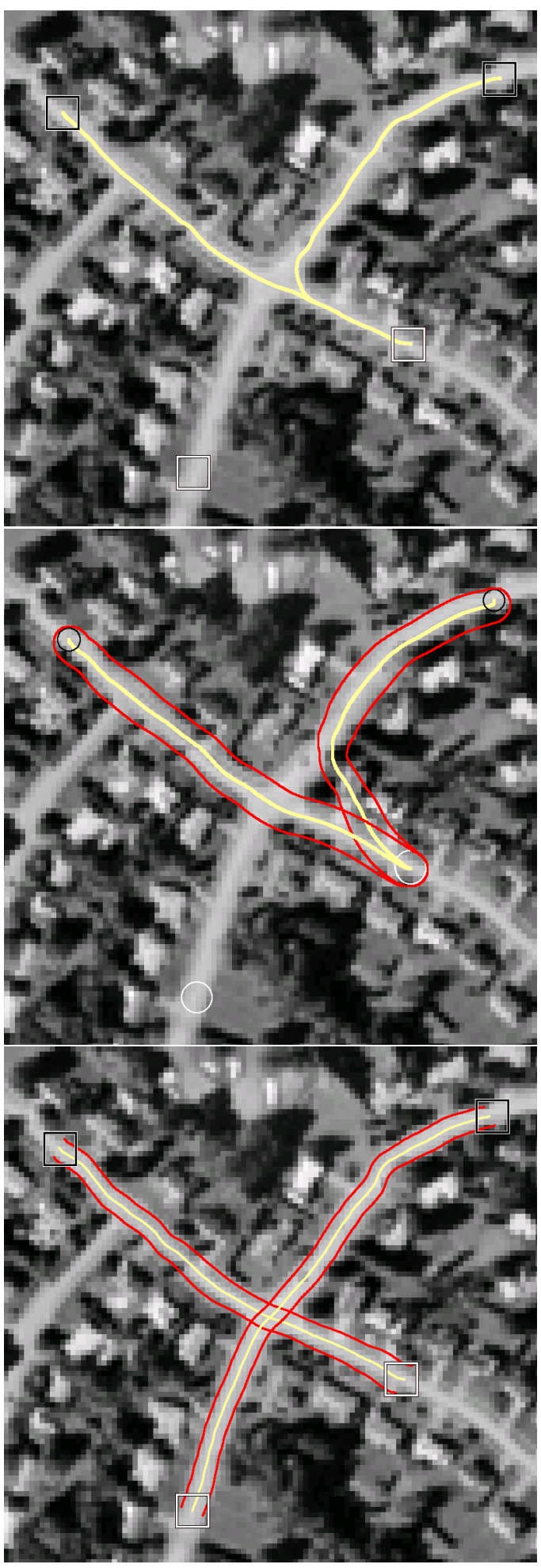

Figure 9. Centerlines and radii extraction of roads for the three tested methods. Two starting point (white squares/circles) and two ending points (black squares/circles) were provided for each method. From top to bottom : classical FM, Li/Yezzi, proposed method. 
[4] L. Cohen. Minimal Paths and Fast Marching Methods for Image Analysis. Nikos Paragios and Yunmei Chen, 2005. 4, 5

[5] L. Cohen and R. Kimmel. Fast marching the global minimum of active contours, 1996. 1

[6] T. Deschamps and L. Cohen. Fast extraction of minimal paths in 3D images and applications to virtual endoscopy. Medical Image Analysis, 5(4):281-299, Dec. 2001. 1

[7] B. Fang, W. Hsu, and M. Lee. Reconstruction of vascular structures in retinal images. In ICIP, volume 2, pages 157160, 2003. 1

[8] A. Hoover, V. Kouznetsova, , and M. Goldbaum. Locating blood vessels in retinal images by piecewise threshold probing of a matched filter response. In Proceedings IEEE Transactions on Medical Imaging, number 19 in 3, pages 203-210. IEEE Computer Society, 2000. 1

[9] H. F. Jelinek and R. M. Cesar-Jr. Segmentation of retinal fundus vasculature in non-mydriatic camera images using wavelets. In J. Suri and T. Laxminarayan, editors, Angiography and Plaque Imaging: Advanced Segmentation Techniques, pages 193-224. CRC Press, 2003. 1

[10] L. Jonasson, X. Bresson, P. Hagmann, J. Thiran, and V. Wedeen. Representing Diffusion MRI in 5D Simplifies Regularization and Segmentation of White Matter Tracts. IEEE Transactions on Medical Imaging, 26:1547-1554, 11 2007. 3

[11] C. Kirbas and F. Quek. A review of vessel extraction techniques and algorithms. Technical report, VisLab Wright State University, Dayton, Ohio, Nov 2000. 1

[12] D. Kleinfeld, P. Mitra, F. Helmchen, and W. Denk. Fluctuations and stimulus-induced changes in blood flow observed in individual capillaries in layers 2 through 4 of rat neocortex. Proceedings of National Academy of Science, USA, 95(26):15741-6, 1998. 2

[13] M. Lalonde, L. Gagnon, and M.-C. Boucher. Non-recursive paired tracking for vessel extraction from retinal images. In Proceedings of the 31st International Symposium on Robotics, pages 61-68, may 2000. 1

[14] H. Li and A. Yezzi. Vessels as 4d curves: Global minimal 4d paths to extract $3 \mathrm{~d}$ tubular surfaces. In $C V P R W^{\prime}$ '06, page 82, Washington, DC, USA, 2006. IEEE Computer Society. 1, 4, 5

[15] M. Niemeijer, J. Staal, B. van Ginneken, M. Loog, and M. Abramoff. Comparative study of retinal vessel segmentation methods on a new publicly available database. In J. M. Fitzpatrick and M. Sonka, editors, SPIE Medical Imaging, volume 5370, pages 648-656. SPIE, SPIE, 2004. 2, 4

[16] K. Poon, G. Hamarneh, and R. Abugharbieh. Live-vessel: Extending livewire for simultaneous extraction of optimal medial and boundary paths in vascular images. In Proceedings of MICCAI (2), pages 444-451, 2007. 1

[17] V. Prinet, O. Monga, C. Ge, S. L. Xie, and S. D. Ma. Thin network extraction in 3d images: Application to medical angiograms. In ICPR '96: Proceedings of the International Conference on Pattern Recognition, volume 3, page 386, Washington, DC, USA, 1996. IEEE Computer Society. 1
[18] P. Pérez, A. Blake, and M. Gangnet. JetStream: probabilistic contour extraction with particles. In Proceedings of IEEE International Conference on Computer Vision, ICCV'01, volume 2, pages 524-531, Vancouver, Canada, July 2001. 1

[19] A. Santamaría-Pang, C. M. Colbert, P. Saggau, and I. A. Kakadiaris. Automatic centerline extraction of irregular tubular structures using probability volumes from multiphoton imaging. In Proceedings of MICCAI (2), pages 486-494, 2007. 1

[20] M. Schaap, R. Manniesing, I. Smal, T. van Walsum, A. van der Lugt, and W. J. Niessen. Bayesian tracking of tubular structures and its application to carotid arteries in cta. In Proceedings of MICCAI (2), pages 562-570, 2007. 1

[21] H. Schmitt, M. Grass, V. Rasche, O. Schramm, S. Hähnel, and K. Sartor. An x-ray based method for the determination of the contrast agent propagation in $3 \mathrm{~d}$ vessel structures. IEEE Transactions on Medical Imaging, 21(3):251262, 2002. 1

[22] J. A. Sethian. Fast marching methods. SIAM Review, 41(2):199-235, 1999. 3

[23] J. A. Sethian. Level Set Methods and Fast Marching Methods: Evolving Interfaces in Computational Geometry, Fluid Mechanics, Computer Vision, and Materials Science. Cambridge University Press, 1999. 3

[24] J. Staal, M. Abramoff, M. Niemeijer, M. Viergever, and B. van Ginneken. Ridge based vessel segmentation in color images of the retina. IEEE Transactions on Medical Imaging, 23(4):501-509, 2004. 4

[25] Y. YANG, S. HUANG, and N. RAO. An automatic hybrid method for retinal blood vessel extraction. International Journal of applied Mathematics and Computer Science, 18(3):399-407, 2008. 1 